Pacific Journal of Mathematics

ON CONTINUOUS APPROXIMATIONS FO 


\title{
ON CONTINUOUS APPROXIMATIONS FOR MULTIFUNCTIONS
}

\author{
F. S. De Blasi and J. MyjaK
}

\begin{abstract}
Problems concerning the approximation of convex valued multifunctions by continuous ones are considered. Approximation results of the type obtained by Gel'man, Cellina, and Hukuhara for Pompeiu-Hausdorff upper semicontinuous multifunctions are shown to hold for some larger classes of multifunctions. Moreover, it is proved that PompeiuHausdorff semicontinuous multifunctions, with convex bounded values, are continuous almost everywhere (in the sense of the Baire category). As an application, an alternative proof is given of Kenderov's theorem stating that a maximal monotone operator is almost everywhere singlevalued.
\end{abstract}

1. Introduction and preliminaries. Let $X$ be a metric space. Let $Y$ be a normed space. Denote by $\mathscr{C}(Y)\left(\right.$ resp. $\left.\mathscr{C}_{b}(Y), \mathscr{C}_{k}(Y)\right)$ the class of all nonempty subsets of $Y$ which are convex (resp. convex bounded, convex compact). In any metric space, $S(u, r)$ stands for the open ball around $u$ with radius $r>0$.

We shall consider the following approximation problems (for the terminology see below):

I. Given a multifunction $F: X \rightarrow \mathscr{C}_{b}(Y)$ and an $\varepsilon>0$, find an $h$-continuous multifunction $G: X \rightarrow \mathscr{C}_{b}(Y)$ such that $h$ (graph $G$, graph $F) \leq \varepsilon$ (where $h$ denotes the Pompeiu-Hausdorff pseudometric).

II. Given a multifunction $F: X \rightarrow \mathscr{C}(Y)$ and an $\varepsilon>0$, find a continuous single-valued function $g: X \rightarrow Y$ such that $h^{*}$ (graph $g$, graph $F$ ) $\leq \varepsilon$ (where $h^{*}$ denotes the separation function).

III. Given a multifunction $F: X \rightarrow \mathscr{C}_{b}(Y)$, find a sequence $\left\{G_{n}\right\}$ of $h$-continuous multifunctions $G_{n}: X \rightarrow \mathscr{C}_{b}(Y)$ satisfying for each $x \in X$, $h\left(G_{n}(x), F(x)\right) \rightarrow 0$ as $n \rightarrow+\infty$, and $G_{n}(x) \supset F\left(S\left(x, \sigma_{n}(x)\right)\right)$ for some $\sigma_{n}(x)>0$.

Apparently, the idea of constructing continuous approximations for a multifunction goes back to Von Neumann [29]. When $F$ is upper semicontinuous in the sense of the Pompeiu-Hausdorff separation $h^{*}$ " $h$ *u.s.c.", the approximation problems I, II, and III have been investigated by Gel'man (see references in [2]), Cellina [5, 6], and Hukuhara [17], respectively. Further results can be found in [23], [24], [13], [8]. 
In this paper, following some ideas of Gel'man, Cellina, and Hukuhara, we shall consider the above approximation problems for more general classes of multifunctions. $\$ 2$ is devoted to the approximation problem $I$. It is shown that such problem is solvable for $F$ in the class of locally convexifying and locally bounded multifunctions. In view of Corollary 2.1, this class seems to be a natural setting for solving problem I. In $\S 3$ the approximation problem II is considered and some results of the type proved by Cellina $[5,6]$, are obtained. In $\$ 4$ we treat the problem III. Furthermore, we characterize some classes of semicontinuous multifunctions by the convergence properties of appropriate $h$-continuous approximations. In $\$ 5$ we give some applications. For example, using an approximation result of $\S 4$, it is proved that each $h^{*}$-u.s.c. multifunction $F: X \rightarrow \mathscr{C}_{b}(Y)$ is $h$-continuous except at points of a Baire first category set (see [15], [22], [7], [12], [20], [21] for similar results). Also we deduce a theorem due to Kenderov [18] stating that a maximal monotone operator is almost everywhere single-valued.

Let us introduce, now, notation and terminology.

Throughout this paper $X$ and $Z$ denote metric spaces, while $Y$ denotes a (real) normed space. The distance function in $X, Z$ is denoted by $d$, and the norm of $Y$ by $\|\cdot\| \cdot 2^{Z}$ (resp. $2^{Y}$ ) stands for the family of all nonempty subsets of $Z$ (resp. $Y$ ). We shall consider the following subsets of $2^{Y}$ :

$$
\begin{aligned}
\mathscr{C}(Y) & =\left\{A \in 2^{Y} \mid A \text { is convex }\right\} \\
\mathscr{C}_{b}(Y) & =\left\{A \in 2^{Y} \mid A \text { is convex bounded }\right\} \\
\mathscr{C}_{k}(Y) & =\left\{A \in 2^{Y} \mid A \text { is convex compact }\right\} .
\end{aligned}
$$

For any $A \subset Y, \operatorname{co} A$ and $\overline{\operatorname{co}} A$ denote respectively the convex hull and the closed convex hull of $A$.

Let $\mathscr{X}$ be an arbitrary metric space, with distance $d$. By $S(x, \sigma)$ we denote the open ball in $\mathscr{X}$ with center at $x$ and radius $\sigma>0$. In a normed space, for notational convenience we set $S=S(0,1)$. For any set $A \subset \mathscr{X}$ we denote by $\bar{A}$ and int $A$ respectively the closure and the interior of $A$.

Given a point $a \in \mathscr{X}$ and a nonempty set $B \subset \mathscr{X}$, we put

$$
r(a, B)=\inf \{d(a, b) \mid b \in B\} .
$$

If $A$ and $B$ are nonempty subsets of $\mathscr{X}$, we define

$$
\begin{aligned}
h^{*}(A, B) & =\sup \{r(a, B) \mid a \in A\}, \\
h(A, B) & =\max \left\{h^{*}(A, B), h^{*}(B, A)\right\} .
\end{aligned}
$$


$h^{*}(A, b)$ is called the (generalized) separation of $A$ from $B$. As it is well known [4, p. 38], $h$ is a (generalized) pseudometric in $2^{\mathscr{X}}$; when $h$ is restricted to the space of the nonempty closed subsets of $\mathscr{X}$, it becomes the Pompeiu-Hausdorff (generalized) metric. In particular, on the space of the nonempty bounded (resp. bounded closed) subsets of a normed space, $h$ is the usual Pompeiu-Hausdorff pseudometric (resp. metric).

For $A \subset \mathscr{X}(A \neq \varnothing)$ and $\varepsilon>0$, we set $N_{\varepsilon}(A)=\{x \in \mathscr{X} \mid r(x, A)<$ $\varepsilon\}$. Observe that if $h^{*}(A, B)<\varepsilon$ (resp. $A \subset N_{\varepsilon}(B)$ ), then $A \subset N_{\varepsilon}(B)$ (resp. $\left.h^{*}(A, B) \leq \varepsilon\right)$. From these it follows easily that $h^{*}(A, B)=$ $\inf \left\{\varepsilon>0 \mid A \subset N_{\varepsilon}(B)\right\}$. Moreover, if $\mathscr{X}$ is a normed space, we have

$$
\begin{aligned}
h^{*}(A, B) & =\inf \{\varepsilon>0 \mid A \subset B+\varepsilon S\}, \\
h(A, B) & =\inf \{\varepsilon>0 \mid A \subset B+\varepsilon S, B \subset A+\varepsilon S\} .
\end{aligned}
$$

Let $\mathscr{X}_{1}, \mathscr{X}_{2}$ be metric spaces with distance functions $d_{1}, d_{2}$. We always assume the Cartesian product $\mathscr{X}_{1} \times \mathscr{X}_{2}$ to be endowed with the metric

$$
e\left(\left(x_{1}, x_{2}\right),\left(x_{1}^{\prime}, x_{2}^{\prime}\right)\right)=\max \left\{d_{1}\left(x_{1}, x_{1}^{\prime}\right), d_{2}\left(x_{2}, x_{2}^{\prime}\right)\right\},
$$

where $\left(x_{1}, x_{2}\right),\left(x_{1}^{\prime}, x_{2}^{\prime}\right) \in \mathscr{X}_{1} \times \mathscr{X}_{2}$.

By a neighborhood of a point $x \in \mathscr{X}$ we mean an open subset of $\mathscr{X}$ containing $x$.

A family $\mathscr{P}=\left\{p_{i}\right\}_{i \in I}$ of continuous functions $p_{i}: X \rightarrow[0,1]$ is called a partition of unity on $X$ if the family $\left\{\operatorname{supp} p_{i}\right\}_{i \in I}$ consisting of the closed sets $\operatorname{supp} p_{i}=\left\{x \in X \mid p_{i}(x)>0\right\}$ is a neighborhood finite closed covering of $X$, and $\sum_{i \in I} p_{i}(x)=1$ for each $x \in X$. We say that a partition $\mathscr{P}$ of unity is subordinated to a given open covering $\left\{U_{i}\right\}_{i \in I}$ of $X$ if, for every $i \in I$, the support of each $p_{\imath}$ lies in the corresponding $U_{i}$. It is well known [9, p. 170] that each open covering of a metric space admits a partition of unity subordinated to it. For any given partition $\mathscr{P}=$ $\left\{p_{\imath}\right\}_{i \in I}$ of unity on $X$ and any $A \subset X$ we set

$$
D_{\mathscr{P}}(A)=\left\{i \in I \mid A \cap \operatorname{supp} p_{i} \neq \varnothing\right\} .
$$

Observe that each $x \in X$ has a neighborhood $V$ such that $D_{\mathscr{P}}(V)$ is finite.

By a multifunction $F: X \rightarrow 2^{Z}$ we mean a mapping $F$ with domain $X$ and range contained in $2^{Z}$. The set

$$
\text { graph } F=\{(x, z) \in X \times Z \mid x \in X \text { and } z \in F(x)\}
$$

is called the graph of $F$. For arbitrary $A \subset X$ and $B \subset Z$ we set

$$
F(A)=\bigcup\{F(x) \mid x \in A\}, \quad F^{-}(B)=\{x \in X \mid F(x) \cap B \neq \varnothing\} .
$$

Note that $F(A)$ is a subset of $Z$. 
We shall review some definitions of upper semicontinuity "u.s.c." and lower semicontinuity "l.s.c." for multifunctions. For the reader's convenience, the definitions are compared in a series of remarks and examples (for the proofs see [1], [16], [26]).

Definition 1.1. $F: X \rightarrow 2^{Z}$ is called u.s.c. if for every set $C$ closed in $Z$ the set $F^{-}(C)$ is closed in $X$.

REMARK 1.1. $F: X \rightarrow 2^{Z}$ is u.s.c. if and only if for every set $V$ open in $Z$ the set $\{x \in X \mid F(x) \subset V\}$ is open in $X$.

Definition 1.2. $F: X \rightarrow 2^{Z}$ is called $h^{*}$-u.s.c. if for every $x_{0} \in X$ and for every $\varepsilon>0$ there is a $\delta>0$ such that $h^{*}\left(F(x), F\left(x_{0}\right)\right)<\varepsilon$ for every $x \in S\left(x_{0}, \delta\right)$.

Definition 1.3. $F: X \rightarrow 2^{Z}$ is called $K$-closed if graph $F$ is a closed set in $X \times Z$.

In the abbreviation " $h *$-u.s.c.", $h^{*}$ is written to emphasize the role of the Pompeiu-Hausdorff (generalized) separation $h^{*}$. In Definition 1.3, $K$ stands for Kuratowski.

REMARK 1.2. $F: X \rightarrow 2^{Z}$ is $K$-closed if and only if, given any two sequences $\left\{x_{n}\right\} \subset X$ and $\left\{z_{n}\right\} \subset Z$ such that $z_{n} \in F\left(x_{n}\right), x_{n} \rightarrow x$, and $z_{n} \rightarrow z$ then we have $z \in F(x)$.

Remark 1.3. Each u.s.c. multifunction $F: X \rightarrow 2^{Z}$ is $h^{*}$-u.s.c.

Example 1.1. Let $F: \mathbf{R} \rightarrow 2^{\mathbf{R}^{2}}$ be defined by $F(x)=\left\{(x, y) \in \mathbf{R}^{2} \mid\right.$ $0 \leq y \leq 1 /|x|\}$ if $x \neq 0$, and $F(0)=\left\{(0, y) \in \mathbf{R}^{2} \mid y \geq 0\right\}$. Clearly $F$ is $h^{*}$-u.s.c. However $F$ is not u.s.c. because while $C=\left\{(1 / n, n) \in \mathbf{R}^{2} \mid n \in\right.$ $\mathbf{N}$ \} is closed in $\mathbf{R}^{2}$, the set $F^{-}(C)$ is not closed in $\mathbf{R}$.

REMARK 1.4. Each closed valued $h^{*}$-u.s.c. multifunction $F: X \rightarrow 2^{Z}$ is $K$-closed.

EXAMPle 1.2. The multifunction $F: \mathbf{R} \rightarrow 2^{\mathbf{R}^{2}}$ defined by $F(x)=$ $\left\{(t, x t) \in \mathbf{R}^{2} \mid t \in \mathbf{R}\right\}$ is $K$-closed but not $h^{*}$-u.s.c.

Remark 1.5. Let $F: X \rightarrow 2^{Z}$ be compact valued. Then $F$ is u.s.c. if and only if $F$ is $h^{*}$-u.s.c.

Remark 1.6. Let $F: X \rightarrow 2^{Z}$ be compact valued. Let $\overline{F(X)} \subset Z$ be compact. Then $F$ is $h^{*}$-u.s.c. if and only if $F$ is $K$-closed. 
Definition 1.4. $F: X \rightarrow 2^{Z}$ is called 1.s.c. if for every set $V$ open in $Z$ the set $F^{-}(V)$ is open in $X$.

Definition 1.5. $F: X \rightarrow 2^{Z}$ is called $h^{*}$-1.s.c. if for every $x_{0} \in X$ and for every $\varepsilon>0$ there is a $\delta>0$ such that $h^{*}\left(F\left(x_{0}\right), F(x)\right)<\varepsilon$ for every $x \in S\left(x_{0}, \delta\right)$.

REMARK 1.7. Each $h^{*}$-1.s.c. multifunction $F: X \rightarrow 2^{Z}$ is 1.s.c.

The multifunction $F$ defined in Example 1.2 is 1.s.c. but not $h^{*}$-1.s.c.

REMARK 1.8. Let $F: X \rightarrow 2^{Z}$ be compact valued. Then $F$ is 1.s.c. if and only if $F$ is $h^{*}$-1.s.c.

Definition 1.6. A multifunction $F: X \rightarrow 2^{Z}$ which is both u.s.c. (resp. $h^{*}$-u.s.c.) and 1.s.c. (resp. $h^{*}$-1.s.c.) is called continuous (resp. $h$-continuous).

REMARK 1.9. Let $F: X \rightarrow 2^{Z}$ be compact valued. In view of Remarks 1.5 and $1.8, F$ is continuous if and only if $F$ is $h$-continuous. In general, neither of these statements implies the other as it is shown in the examples below.

Example 1.3. The multifunction $F: \mathbf{R} \rightarrow 2^{\mathbf{R}}$ defined by $F(x)=$ $[-1 /|x|, 1 /|x|]$ if $x \neq 0$, and $f(0)=\mathbf{R}$, is continuous but not $h$-continuous.

EXAmPLE 1.4. Let $c_{0}$ be the Banach space of all real sequences $z=\left(z_{1}, z_{2}, \ldots\right)$ such that $z_{n} \rightarrow 0$, with norm $\|z\|=\sup \left\{\left|z_{n}\right| \mid n \in \mathbf{N}\right\}$. Denote by $A \subset c_{0}$ a closed bounded convex body with the following property: there exists a point $\tilde{z} \in c_{0} \backslash A$ such that $\|\tilde{z}-a\|>r(\tilde{z}, A)$ for each $a \in A$. The existence of such a set $A$ follows from [11]. Define $F$ : $\mathbf{R} \rightarrow 2^{c_{0}}$ by $F(x)=A$ if $x \leq 0$, and $F(x)=\overline{A+x S}$ if $x>0$. Clearly $F$ is a (closed valued) $h$-continuous multifunction. On the other hand for $B=\left\{z \in c_{0} \mid\|z-\tilde{z}\| \leq r(\tilde{z}, A)\right\}$, a closed subset of $c_{0}$, we have $F^{-}(B)$ $=(0,+\infty)$, which is not closed in R. Hence $F$ is not u.s.c. and so not even continuous.

Definition 1.7. $F: X \rightarrow 2^{Z}$ is called weakly $h^{*}$-u.s.c. at $x_{0}$ if for every $\varepsilon>0$ and $\eta>0$ there is a $\delta(0<\delta \leq \eta)$ and there is a point 
$x^{\prime} \in S\left(x_{0}, \delta\right)$ such that $h^{*}\left(F(x), F\left(x^{\prime}\right)\right)<\varepsilon$ for every $x \in S\left(x_{0}, \delta\right) . F$ is called weakly $h^{*}$-u.s.c. if it is weakly $h^{*}$-u.s.c. at each $x_{0} \in X$.

If $x^{\prime}=x_{0}$ the above definition reduces to that of an $h^{*}$-u.s.c. multifunction. While each $h^{*}$-u.s.c. multifunction is weakly $h^{*}$-u.s.c., the converse is not true in general.

It is easy to see that a weakly $h^{*}$-u.s.c. multifunction is not necessarily $K$-closed. The next example shows that a $K$-closed multifunction can fail to be weakly $h^{*}$-u.s.c.

EXAMPLE 1.5. Let $X$ be the set of the rational numbers with the usual metric. Order $X$ into a sequence $\left\{x_{1}, x_{2}, \ldots\right\}$. Define $F: X \rightarrow 2^{\mathbf{R}}$ by $F\left(x_{n}\right)=\{n\}$. Clearly $F$ is $K$-closed but not weakly $h^{*}$-u.s.c.

Let $f: X \rightarrow Z$ be a single-valued function. Then $f$ is u.s.c. (resp. 1.s.c., $h^{*}$-u.s.c., $h^{*}$-1.s.c., weakly $h^{*}$-u.s.c.) if and only if $f$ is continuous.

DEFINITION 1.8. $F: X \rightarrow 2^{Z}$ is called locally bounded if for each $x \in X$ there exists a $\delta>0$ such that $F(S(x, \delta))$ is a bounded subset of $Z$.

DEFinition 1.9. $F: X \rightarrow 2^{Y}$ is called locally convexifying if for every $x \in X$ and for every $\varepsilon>0$ and $\eta>0$, there is a $\sigma(x), 0<\sigma(x) \leq \eta$, such that $\operatorname{co} F(S(x, \sigma(x))) \subset F(S(x, \sigma(x)))+\varepsilon S$.

Each weakly $h^{*}$-u.s.c. multifunction $F: X \rightarrow \mathscr{C}_{b}(Y)$ is locally convexifying and locally bounded. The converse is not true in general. Moreover a locally convexifying and locally bounded multifunction is not necessarily $K$-closed.

REMARK. 1.10. Some of the above definitions and remarks, in particular Definitions 1.1, 1.3, 1.4, and Remark 1.1, are meaningful also for multifunctions from a topological space to the nonempty subsets of another topological space.

2. Continuous multi-valued approximations for multifunctions. In this section we consider the problem of approximating a multifunction with convex bounded values by another one which is $h$-continuous.

THEOREM 2.1. Let $F: X \rightarrow \mathscr{C}_{b}(Y)$ be locally convexifying and locally bounded. Then for every $\varepsilon>0$ there is an h-continuous multifunction $G$ : $X \rightarrow \mathscr{C}_{b}(Y)$ with the following properties:

(i) for each $x \in X$ there is a $\sigma(x)>0$ such that $F(S(x, \sigma(x))) \subset G(x)$;

(ii) $h$ (graph $G$, graph $F$ ) $\leq \varepsilon$. 
Proof. Let $\varepsilon>0$. Let $z \in X$. Since $F$ is locally bounded and locally convexifying, there is a $\delta_{z}=\delta(z, \varepsilon)\left(0<\delta_{z}<\varepsilon / 2\right)$ such that $F\left(S\left(z, \delta_{z}\right)\right)$ is bounded and

$$
\operatorname{co} F\left(S\left(z, \delta_{z}\right)\right) \subset F\left(S\left(z, \delta_{z}\right)\right)+\varepsilon S .
$$

Evidently $\mathscr{V}=\left\{S\left(z, \delta_{z} / 3\right)\right\}_{z \in X}$ is an open covering of $X$. Denote by $\mathscr{P}=\left\{p_{z}\right\}_{z \in X}$ a partition of unity subordinated to $\mathscr{V}$. For each $x \in X$, set

$$
G(x)=\sum_{z \in X} p_{z}(x) G_{z} \quad \text { where } G_{z}=\operatorname{co} F\left(S\left(z, \delta_{z} / 3\right)\right) .
$$

It is routine to verify that $(2.1)$ defines an $h$-continuous multifunction $G$ : $X \rightarrow \mathscr{C}_{b}(Y)$.

Let $\tilde{x} \in X$. Let $D_{\mathscr{P}}(\tilde{x})=\left\{z_{1}, z_{2}, \ldots, z_{k}\right\}$. We have

$$
G(\tilde{x})=\sum_{i=1}^{k} p_{z_{i}}(\tilde{x}) G_{z_{i}}
$$

Since $\tilde{x} \in \operatorname{supp} p_{z_{1}} \subset S\left(z_{i}, \delta_{z_{i}} / 3\right)(i=1,2, \ldots, k)$, there exists a $\sigma=\sigma(\tilde{x})$ $>0$ such that $S(\tilde{x}, \sigma) \subset S\left(z_{i}, \delta_{z_{i}} / 3\right)$ for each $i=1,2, \ldots, k$. Then we have

$$
F(S(\tilde{x}, \boldsymbol{\sigma})) \subset \sum_{i=1}^{k} p_{z_{i}}(\tilde{x}) F(S(\tilde{x}, \boldsymbol{\sigma})) \subset \sum_{i=1}^{k} p_{z_{i}}(\tilde{x}) G_{z_{l}}=G(\tilde{x})
$$

and so, since $\tilde{x}$ is arbitrary in $X$, (i) is fulfilled.

From (i) it follows that graph $F \subset$ graph $G$. Therefore to prove (ii) it is sufficient to show that $h^{*}$ (graph $G$, graph $\left.F\right) \leq \varepsilon$. To this end, let $(\tilde{x}, \tilde{y}) \in$ graph $G$. Set $D_{\mathscr{P}}(\tilde{x})=\left\{z_{1}, z_{2}, \ldots, z_{k}\right\}$ and $\delta_{z_{0}}=$ $\max \left\{\delta_{z_{1}}, \delta_{z_{2}}, \ldots, \delta_{z_{k}}\right\}$. Since $S\left(z_{i}, \delta_{z_{i}} / 3\right) \subset S\left(z_{i_{0}}, \delta_{z_{t_{0}}}\right)$, we have $G_{z_{l}} \subset$ $\operatorname{co} F\left(S\left(z_{t_{0}}, \delta_{z_{t_{0}}}\right)\right)$ and so

$$
\begin{aligned}
G(\tilde{x}) & =\sum_{i=1}^{k} p_{z_{\imath}}(\tilde{x}) G_{z_{\imath}} \subset \sum_{i=1}^{k} p_{z_{\imath}}(\tilde{x}) \operatorname{co} F\left(S\left(z_{\iota_{0}}, \delta_{z_{t_{0}}}\right)\right) \\
& =\operatorname{co} F\left(S\left(z_{i_{0}}, \delta_{z_{\iota_{0}}}\right)\right) \subset F\left(S\left(z_{\iota_{0}}, \delta_{z_{\iota_{0}}}\right)\right)+\varepsilon S .
\end{aligned}
$$

But $\tilde{y} \in G(\tilde{x})$ thus, for some $x^{\prime} \in S\left(z_{i_{0}}, \delta_{z_{i_{0}}}\right)$ and some $u \in \varepsilon S$, we have $\tilde{y}=y^{\prime}+u$, where $y^{\prime} \in F\left(x^{\prime}\right)$. Clearly,

$$
d\left(\tilde{x}, x^{\prime}\right) \leq d\left(\tilde{x}, z_{i_{0}}\right)+d\left(z_{i_{0}}, x^{\prime}\right)<\delta_{z_{t_{0}}} / 3+\delta_{z_{t_{0}}}<\varepsilon
$$

and $\left\|\tilde{y}-y^{\prime}\right\|<\varepsilon$. Therefore $r((\tilde{x}, \tilde{y})$, graph $F)<\varepsilon$, for $\left(x^{\prime}, y^{\prime}\right)$ lies in graph $F$. Since $(\tilde{x}, \tilde{y})$ is arbitrary in graph $G$, it follows that $h^{*}$ (graph $G$, graph $F$ ) $\leq \varepsilon$. This completes the proof. 
REMARK 2.1. In addition to the hypotheses of Theorem 2.1, suppose that $F$ is compact (that is whenever $B \subset X$ is bounded, the set $F(B)$ is contained in a compact convex subset of $Y$ ). Then, arguing as before one can prove the existence of a compact $h$-continuous multifunction $G$ : $X \rightarrow \mathscr{C}_{k}(Y)$ satisfying the properties (i) and (ii) of Theorem 2.1.

TheOREM 2.2. Let $F: X \rightarrow \mathscr{C}_{k}(Y)$ satisfy at each point $x \in X$ the condition:

(2.2) $\lim _{\delta \rightarrow 0+} h(F(S(x, \delta)), A(x))=0, \quad$ where $A(x)=\bigcap_{\rho>0} \overline{F(S(x, \rho))}$.

In addition, suppose that for every $\varepsilon>0$ there exists an h-continuous multifunction $G: X \rightarrow \mathscr{C}_{k}(Y)$ satisfying the conditions (i) and (ii) of Theorem 2.1. Then $F$ is locally convexifying.

Proof. For a contradiction, suppose that $F$ is not locally convexifying. There exist then an $\tilde{x} \in X$, an $\varepsilon>0$, and a decreasing sequence $\left\{\delta_{n}\right\}$ of positive numbers $\delta_{n}$ converging to zero, such that

$$
\operatorname{co} F\left(S\left(\tilde{x}, \delta_{n}\right)\right) \not \subset F\left(S\left(\tilde{x}, \delta_{n}\right)\right)+3 \varepsilon S, \quad n=1,2, \ldots .
$$

Set $\tilde{A}=A(\tilde{x})$. By (2.2) we have that $h\left(F\left(S\left(\tilde{x}, \delta_{n}\right)\right), \tilde{A}\right) \rightarrow 0$ as $n \rightarrow+\infty$, and so also $h\left(\operatorname{co} F\left(S\left(\tilde{x}, \delta_{n}\right)\right)\right.$, $\left.\operatorname{co} \tilde{A}\right) \rightarrow 0$ as $n \rightarrow+\infty$. Hence, $\tilde{A} \subset$ $F\left(S\left(\tilde{x}, \delta_{n}\right)\right)+(\varepsilon / 2) S$ and $\operatorname{co} F\left(S\left(\tilde{x}, \delta_{n}\right)\right) \subset \operatorname{co} \tilde{A}+(\varepsilon / 2) S$ are satisfied for some $n$ large enough. We have

$$
\operatorname{co} \tilde{A} \not \subset \tilde{A}+2 \varepsilon S \text {. }
$$

In fact, in the contrary case $\operatorname{co} \tilde{A} \subset \tilde{A}+2 \varepsilon S$, and thus

$$
\operatorname{co} F\left(S\left(\tilde{x}, \delta_{n}\right)\right) \subset \operatorname{co} \tilde{A}+(\varepsilon / 2) S \subset \tilde{A}+(5 / 2) \varepsilon S \subset F\left(S\left(\tilde{x}, \delta_{n}\right)\right)+3 \varepsilon S,
$$

a contradiction to (2.3). Let $\tilde{y} \in \operatorname{co} \tilde{A}$ be such that $\tilde{y} \notin \tilde{A}+2 \varepsilon S$. Evidently, $S(\tilde{y}, \varepsilon) \cap(\tilde{A}+\varepsilon S)=\varnothing$. Fix $n_{0} \in \mathbf{N}$ such that $F\left(S\left(\tilde{x}, \delta_{n_{0}}\right)\right) \subset \tilde{A}$ $+\varepsilon S$ and observe that

$$
S(\tilde{y}, \varepsilon) \cap F\left(S\left(\tilde{x}, \delta_{n_{0}}\right)\right)=\varnothing .
$$

Set $\theta=\min \left\{\varepsilon, \boldsymbol{\delta}_{n_{0}}\right\}$. Let $(x, y)$ be an arbitrary point in graph $F$. Since $e((\tilde{x}, \tilde{y}),(x, y)) \geq\|\tilde{y}-y\| \geq \varepsilon \geq \theta$ if $x \in S\left(\tilde{x}, \delta_{n_{0}}\right)$, and $e((\tilde{x}, \tilde{y}),(x, y))$ $\geq d(\tilde{x}, x) \geq \delta_{n_{0}} \geq \theta$ if $x \notin S\left(\tilde{x}, \delta_{n_{0}}\right)$, we have

$$
r((\tilde{x}, \tilde{y}), \operatorname{graph} F) \geq \theta .
$$

On the other hand, by hypothesis there is an $h$-continuous multifunction $G: X \rightarrow \mathscr{C}_{k}(Y)$ which satisfies the condition (i) of Theorem 2.1, and is such that

$$
h(\text { graph } G, \text { graph } F) \leq \theta / 2 \text {. }
$$


For some $\sigma(\tilde{x})>0$ we have $F(S(\tilde{x}, \sigma(\tilde{x}))) \subset G(\tilde{x})$ and so $F\left(S\left(\tilde{x}, \delta_{n}\right)\right) \subset$ $G(\tilde{x})$, provided $n$ is large enough. Since $\tilde{y} \in \operatorname{co} \tilde{A} \subset \operatorname{co} \overline{F\left(S\left(\tilde{x}, \delta_{n}\right)\right)} \subset$ $G(\tilde{x})$, it follows that $(\tilde{x}, \tilde{y}) \in$ graph $G$. Hence by (2.4) we obtain $h^{*}($ graph $G$, graph $F) \geq \theta$, and thus $h$ (graph $G$, graph $\left.F\right) \geq \theta$, a contradiction to (2.5). Therefore $F$ is locally convexifying. This completes the proof.

Proposition 2.1. Each compact multifunction $F: X \rightarrow \mathscr{C}_{k}(Y)$ satisfies the condition (2.2).

Proof. Let $x_{0} \in X$. It suffices to prove that $h^{*}\left(F\left(S\left(x_{0}, \delta\right)\right), A_{0}\right) \rightarrow 0$ as $\delta \rightarrow 0$, where $A_{0}=A\left(x_{0}\right)$. If this is not true, there exists an $\varepsilon>0$ and two sequences $\left\{x_{n}\right\} \subset X$ and $\left\{y_{n}\right\} \subset Y$ such that $x_{n} \rightarrow x_{0}, y_{n} \in F\left(x_{n}\right)$, and $r\left(y_{n}, A_{0}\right)>\varepsilon(n \in \mathbf{N})$. By the compactness of $F$ we assume (without loss of generality) that $y_{n} \rightarrow y_{0} \in Y$, and so $r\left(y_{0}, A_{0}\right) \geq \varepsilon$. On the other hand, given any $\rho>0$, for $n \in \mathbf{N}$ large enough we have $x_{n} \in S\left(x_{0}, \rho\right)$, which implies that $y_{n} \in F\left(S\left(x_{0}, \rho\right)\right)$. Consequently $y_{0} \in \overline{F\left(S\left(x_{0}, \rho\right)\right)}$. Since $\rho>0$ is arbitrary, it follows that $y_{0} \in A_{0}$, a contradiction. This completes the proof.

From Remark 2.1, Proposition 2.1, and Theorem 2.2 we have:

Corollary 2.1. Let $F: X \rightarrow \mathscr{C}_{k}(Y)$ be compact. Then the following statements are equivalent:

(a) $F$ is locally convexifying.

(b) For every $\varepsilon>0$ there exists a (compact) $h$-continuous multifunction $G: X \rightarrow \mathscr{C}_{k}(Y)$ satisfying the conditions (i) and (ii) of Theorem 2.1.

For the next approximation theorem we need the following

LeMma 2.1. Let $F: X \rightarrow \mathscr{C}_{b}(Y)$ be locally convexifying. Suppose that $G_{1}: X \rightarrow \mathscr{C}_{b}(Y)$ is a closed valued h-continuous multifunction satisfying the property: for each $x \in X$ there exists $a \sigma_{1}(x)>0$ and $a \theta_{1}(x)>0$ such that

$$
\operatorname{co} F\left(S\left(x, \sigma_{1}(x)\right)\right)+\theta_{1}(x) S \subset G_{1}(x) .
$$

Then for every $\varepsilon>0$ there exists a closed valued $h$-continuous multifunction $G_{2}: X \rightarrow \mathscr{C}_{b}(Y)$ with the following properties:

that

(i) for every $x \in X$ there exists $a \sigma_{2}(x)>0$ and $a \theta_{2}(x)>0$ such

$$
\operatorname{co} F\left(S\left(x, \sigma_{2}(x)\right)\right)+\theta_{2}(x) S \subset G_{2}(x) ;
$$

(ii) $G_{2}(x) \subset G_{1}(x)$ for each $x \in X$;

(iii) $h$ (graph $G_{2}$, graph $F$ ) $\leq \varepsilon$. 
Proof. Let $\varepsilon>0$. Let $z \in X$. From the hypothesis, there exists a $\sigma_{1}(z)>0$ and $\theta_{1}(z)>0$ such that $\operatorname{co} F\left(S\left(z, \sigma_{1}(z)\right)\right)+\theta_{1}(z) S \subset G_{1}(z)$. By the $h$-continuity of $G_{1}$, there exists a $\rho_{z}, 0<\rho_{z} \leq \sigma_{1}(z)$, such that $G_{1}(z)$ $\subset G_{1}(x)+\frac{1}{2} \theta_{1}(z) S$ for every $x \in S\left(z, \rho_{z}\right)$. Hence, $\operatorname{co} F\left(S\left(z, \sigma_{1}(z)\right)\right)+$ $\theta_{1}(z) S \subset G_{1}(x)+\frac{1}{2} \theta_{1}(z) S$ and so, since $G_{1}(x)$ is a convex closed set, by Rådström lemma [27] we have

(2.6) $\operatorname{co} F\left(S\left(z, \sigma_{1}(z)\right)\right)+\frac{1}{2} \theta_{1}(z) S \subset G_{1}(x)$ for every $x \in S\left(z, \rho_{z}\right)$.

On the other hand $F$ is locally convexifying, thus there is a $\delta_{z}, 0<\delta_{z} \leq$ $\min \left\{\rho_{z}, \varepsilon / 2\right\}$, such that

$$
\operatorname{co} F\left(S\left(z, \delta_{z}\right)\right) \subset F\left(S\left(z, \delta_{z}\right)\right)+(\varepsilon / 2) S .
$$

Set $\theta_{z}=\min \left\{\frac{1}{2} \theta_{1}(z), \varepsilon\right\}$. From (2.6) it follows that

$$
\operatorname{co} F\left(S\left(z, \delta_{z}\right)\right)+\theta_{z} S \subset G_{1}(z) \text { for every } x \in S\left(z, \delta_{z}\right),
$$

because $\delta_{z} \leq \rho_{z} \leq \sigma_{1}(z)$ and $\theta_{z} \leq \frac{1}{2} \theta_{1}(z)$. Let $\mathscr{P}=\left\{p_{z}\right\}_{z \in X}$ be a partition of unity subordinated to the open covering $\mathscr{V}=\left\{S\left(z, \delta_{z} / 3\right)\right\}_{z \in X}$ of $X$. For each $x \in X$, we put

$$
\begin{aligned}
& G_{2}(x)=\overline{\sum_{z \in X} p_{z}(x) G_{z}} \text { where } G_{z}=\operatorname{co} F\left(S\left(z, \frac{\delta_{z}}{3}\right)\right)+\frac{1}{4} \theta_{z} S \\
& \theta_{2}(x)=\frac{1}{4} \sum_{z \in X} p_{z}(x) \theta_{z} .
\end{aligned}
$$

It is routine to see that the above formulas define respectively a closed valued $h$-continuous multifunction $G_{2}: X \rightarrow \mathscr{C}_{b}(Y)$ and a positive continuous function $\theta_{2}: X \rightarrow[0, \varepsilon / 4]$.

Let us show that $G_{2}$ satisfies (i)-(iii). Let $\tilde{x} \in X$. Let $D_{\mathscr{P}}(\tilde{x})=$ $\left\{z_{1}, z_{2}, \ldots, z_{k}\right\}$. We have

$$
G_{2}(\tilde{x})=\overline{\sum_{i=1}^{k} p_{z_{i}}(\tilde{x}) G_{z_{i}}} \quad \theta_{2}(\tilde{x})=\frac{1}{4} \sum_{i=1}^{k} p_{z_{i}}(\tilde{x}) \theta_{z_{i}} .
$$

(i) Indeed, $\tilde{x} \in \operatorname{supp} p_{z_{l}}(\tilde{x}) \subset S\left(z_{i}, \delta_{z_{i}} / 3\right)(i=1,2, \ldots, k)$, thus there is a $\sigma_{2}(\tilde{x})>0$ such that $S\left(\tilde{x}, \sigma_{2}(\tilde{x})\right) \subset S\left(z_{i}, \delta_{z_{i}} / 3\right)$ for each $i=1,2, \ldots, k$. From this it follows

$$
\operatorname{co} F\left(S\left(\tilde{x}, \sigma_{2}(\tilde{x})\right)\right) \subset \sum_{i=1}^{k} p_{z_{l}}(\tilde{x}) \operatorname{co} F\left(S\left(z_{\imath}, \frac{\delta_{z_{l}}}{3}\right)\right)
$$

and so

$$
\begin{aligned}
\operatorname{co} F\left(S\left(\tilde{x}, \sigma_{2}(\tilde{x})\right)\right)+\theta_{2}(\tilde{x}) S & \subset \sum_{i=1}^{k} p_{z_{l}}(\tilde{x})\left[\operatorname{co} F\left(S\left(z_{i}, \frac{\delta_{z_{t}}}{3}\right)\right)+\frac{1}{4} \theta_{z_{i}} S\right] \\
& \subset G_{2}(\tilde{x}) .
\end{aligned}
$$


Since $\tilde{x}$ is arbitrary in $X$, (i) is proved.

(ii) For each $i=1,2, \ldots, k$ we have $\tilde{x} \in S\left(z_{i}, \delta_{z_{1}} / 3\right)$ and hence, by (2.8), $G_{z_{l}} \subset G_{1}(\tilde{x})$. Therefore

$$
\sum_{i=1}^{k} p_{z_{i}}(\tilde{x}) G_{z_{\iota}} \subset \sum_{i=1}^{k} p_{z_{i}}(\tilde{x}) G_{1}(\tilde{x})=G_{1}(\tilde{x}),
$$

from which $G_{2}(\tilde{x}) \subset G_{1}(\tilde{x})$ follows at once.

(iii) In view of (i), it suffices to show that $h^{*}$ (graph $G_{2}$, graph $\left.F\right) \leq \varepsilon$. Indeed, let $(\tilde{x}, \tilde{y}) \in$ graph $G_{2}$. Set $D_{\mathscr{P}}(\tilde{x})=\left\{z_{1}, z_{2}, \ldots, z_{k}\right\}$ and let $\delta_{z_{t_{0}}}=$ $\max \left\{\delta_{z_{1}}, \delta_{z_{2}}, \ldots, \delta_{z_{k}}\right\}$. Since $S\left(z_{i}, \delta_{z_{i}} / 3\right) \subset S\left(z_{i_{0}}, \delta_{z_{z_{0}}}\right)$, we have $\operatorname{co} F\left(S\left(z_{i}, \delta_{z_{t}} / 3\right)\right) \subset \operatorname{co} F\left(S\left(z_{t_{0}}, \delta_{z_{t_{0}}}\right)\right)$. Using this inclusion in the formula giving $G_{2}(\tilde{x})$ (see (2.9)), one easily finds

$$
G_{2}(\tilde{x}) \subset \overline{\operatorname{co} F\left(S\left(z_{\iota_{0}}, \delta_{z_{\iota_{0}}}\right)\right)+\theta_{2}(\tilde{x}) S} .
$$

Hence, by (2.7),

$$
G_{2}(\tilde{x}) \subset \overline{F\left(S\left(z_{i_{0}}, \delta_{z_{t_{0}}}\right)\right)+(\varepsilon / 2) S+\theta_{2}(\tilde{x}) S}
$$

and so $G_{2}(\tilde{x}) \subset F\left(S\left(z_{i_{0}}, \delta_{z_{t_{0}}}\right)\right)+\varepsilon S$, because $\theta_{2}(\tilde{x}) \leq \varepsilon / 4$. Then, as in the proof of Theorem 2.1, one shows that $r((\tilde{x}, \tilde{y})$, graph $F)<\varepsilon$. Since $(\tilde{x}, \tilde{y})$ is arbitrary in graph $G_{2}$, we infer that $h^{*}\left(\right.$ graph $G_{2}$, graph $\left.F\right) \leq \varepsilon$. This completes the proof.

THEOREM 2.3. Let $F: X \rightarrow \mathscr{C}_{b}(Y)$ be locally convexifying and locally bounded. Then there is a sequence $\left\{G_{n}\right\}$ of closed valued h-continuous multifunctions $G_{n}: X \rightarrow \mathscr{C}_{b}(Y)$ with the following properties:

(i) for every $n \in \mathbf{N}$ and $x \in X$ there is a $\sigma_{n}(x)>0$ such that $F\left(S\left(x, \sigma_{n}(x)\right)\right) \subset G_{n}(x)$;

(ii) $G_{1}(x) \supset G_{2}(x) \supset \ldots$, for each $x \in X$;

(iii) $h$ (graph $G_{n}$, graph $\left.F\right) \rightarrow 0$ as $n \rightarrow+\infty$.

Proof. Set $\varepsilon_{n}=1 / n, n \in \mathbf{N}$. By Theorem 2.1 there is an $h$-continuous multifunction $G: X \rightarrow \mathscr{C}_{b}(Y)$ with the following two properties: $h$ (graph $G$, graph $F$ ) $\leq 1 / 2$; for each $x \in X$ there is a $\sigma_{1}(x)>0$ such that $F\left(S\left(x, \sigma_{1}(x)\right)\right) \subset G(x)$. Define $G_{1}: X \rightarrow \mathscr{C}_{b}(Y)$ by $G_{1}(x)=\overline{G(x)+(1 / 2) S}$. Observe that: $G_{1}$ is closed valued and $h$-continuous; $h$ (graph $G_{1}$, graph $\left.F\right) \leq \varepsilon_{1}$; and $\operatorname{co} F\left(S\left(x, \sigma_{1}(x)\right)\right)+(1 / 2) S \subset$ $G_{1}(x)$ for each $x \in X$. Since $F$ and $G_{1}$ satisfy the hypotheses of Lemma 2.1 (with $\theta_{1}(x) \equiv 1 / 2$ ), there is a closed valued $h$-continuous multifunction $G_{2}: X \rightarrow \mathscr{C}_{b}(Y)$ with the following properties: $h$ (graph $G_{2}$, graph $F) \leq \varepsilon_{2} ; G_{2}(x) \subset G_{1}(x)$ for each $x \in X$; for every $x \in X$ there 
exist a $\sigma_{2}(x)>0$ and a $\theta_{2}(x)>0$ such that $\operatorname{co} F\left(S\left(x, \sigma_{2}(x)\right)\right)+\theta_{2}(x) S$ $\subset G_{2}(x)$. By induction, using Lemma 2.1, one constructs a sequence $\left\{G_{n}\right\}$ of closed valued $h$-continuous multifunctions $G_{n}: X \rightarrow \mathscr{C}_{b}(Y)$ enjoying the properties (i)-(iii) of the theorem. This completes the proof.

3. Continuous single-valued approximations for multifunctions. In this section we treat the problem of approximating a multifunction with convex values by a single-valued continuous function.

LeMma 3.1. Let $F: X \rightarrow 2^{Y}$ be an arbitrary multifunction. Let $\delta$ : $X \rightarrow \mathbf{R}$ be a l.s.c. positive function. Then the multifunction $G: X \rightarrow \mathscr{C}(Y)$ defined by $G(x)=\operatorname{co} F(S(x, \delta(x)))$ is l.s.c.

Proof. Let $V$ be open in $Y$. We claim that the set $U=\{x \in X \mid G(x)$ $\cap V \neq \varnothing\}$ is open in $X$. In fact, let $x_{0} \in U$ (the case $U=\varnothing$ is trivial). Fix any point $y_{0} \in G\left(x_{0}\right) \cap V$. Evidently $y_{0}=\lambda_{1} y_{1}+\lambda_{2} y_{2}+\cdots+\lambda_{k} y_{k}$ for suitable points $y_{l} \in F\left(x_{l}\right), x_{i} \in S\left(x_{0}, \delta\left(x_{0}\right)\right)$, and $\lambda_{i}>0$ with $\lambda_{1}+$ $\lambda_{2}+\cdots+\lambda_{k}=1$. Set

$$
\eta=\min \left\{\delta\left(x_{0}\right)-d\left(x_{i}, x_{0}\right) \mid i=1,2, \ldots, k\right\},
$$

and observe that $\eta>0$. Since $\delta$ is l.s.c. there is a $\rho, 0<\rho \leq \eta / 2$, such that $\delta(x)>\delta\left(x_{0}\right)-\eta / 2$ for every $x \in S\left(x_{0}, \rho\right)$. Let $x \in S\left(x_{0}, \rho\right)$ be arbitrary. For each $i=1,2, \ldots, k$ we have $d\left(x_{i}, x_{0}\right) \leq \delta\left(x_{0}\right)-\eta$ and so

$$
\begin{aligned}
d\left(x_{l}, x\right) & \leq d\left(x_{l}, x_{0}\right)+d\left(x_{0}, x\right)<\delta\left(x_{0}\right)-\eta+\rho \\
& \leq \delta\left(x_{0}\right)-\eta / 2<\delta(x),
\end{aligned}
$$

that is $x_{i} \in S(x, \delta(x))$. Therefore $y_{i} \in F\left(x_{i}\right) \subset \operatorname{co} F(S(x, \delta(x)))$ and consequently $y_{0} \in G(x)$. But $y_{0}$ lies in $V$ too, thus $G(x) \cap V \neq \varnothing$. Hence $x \in U$. Since $x$ is arbitrary in $S\left(x_{0}, \rho\right)$, it follows that $S\left(x_{0}, \rho\right) \subset U$. This implies that $U$ is an open set, for $x_{0}$ also is arbitrary in $U$. This completes the proof.

Let $F: X \rightarrow 2^{Y}$. Let $\varepsilon>0$. Define $\Delta(x)=\{0<\rho \leq \varepsilon \mid$ there is an $x^{\prime} \in S(x, \rho)$ such that $h^{*}\left(F(z), F\left(x^{\prime}\right)\right)<\varepsilon$ for every $\left.z \in S(x, \rho)\right\}$. Observe that $\Delta(x)$ is certainly nonempty if $F$ is weakly $h^{*}$-u.s.c.

Lemma 3.2. Let $F: X \rightarrow 2^{Y}$ be weakly $h^{*}$-u.s.c. Let $\varepsilon>0$. Then the function $\delta: X \rightarrow[0, \varepsilon]$ given by $\delta(x)=\sup \Delta(x)$ is positive and l.s.c.

Proof. It is only needed to show that $\delta$ is 1.s.c. To see that, fix an $x_{0} \in X$ and let $\eta, 0<\eta<\delta\left(x_{0}\right)$, be any. Take $\rho \in \Delta\left(x_{0}\right)$ such that 
$\delta\left(x_{0}\right)-\eta / 2<\rho \leq \delta\left(x_{0}\right)$. Then there is an $x^{\prime} \in S\left(x_{0}, \rho\right)$ such that

$$
h^{*}\left(F(z), F\left(x^{\prime}\right)\right)<\varepsilon \quad \text { for each } z \in S\left(x_{0}, \rho\right) .
$$

Set $\sigma=\min \left\{\left(\rho-d\left(x^{\prime}, x_{0}\right)\right) / 2, \eta / 2\right\}$. For each $x \in S\left(x_{0}, \sigma\right)$ we have

$$
x^{\prime} \in S(x, \rho-\sigma) \subset S\left(x_{0}, \rho\right) \text {. }
$$

Indeed, let $x \in S\left(x_{0}, \sigma\right)$. Since $d\left(x^{\prime}, x\right) \leq d\left(x^{\prime}, x_{0}\right)+d\left(x_{0}, x\right)<$ $d\left(x^{\prime}, x_{0}\right)+\sigma$ and $d\left(x^{\prime}, x_{0}\right) \leq \rho-2 \sigma$, it follows that $d\left(x^{\prime}, x\right)<\rho-\sigma$ and so $x^{\prime} \in S(x, \rho-\sigma)$. Furthermore each $z \in S(x, \rho-\sigma)$ satisfies

$$
d\left(z, x_{0}\right) \leq d(z, x)+d\left(x, x_{0}\right)<(\rho-\sigma)+\sigma=\rho,
$$

and hence $z \in S\left(x_{0}, \rho\right)$ which completes the proof of (3.2).

Now take any $x \in S\left(x_{0}, \sigma\right)$. Evidently by (3.2) it follows that (3.1) is satisfied with $S(x, \rho-\sigma)$ in the place of $S\left(x_{0}, \rho\right)$ ( $x^{\prime}$ unchanged). Hence $\rho-\sigma \in \Delta(x)$. Then $\delta(x) \geq \rho-\sigma \geq \rho-\eta / 2$ and so $\delta(x)>\delta\left(x_{0}\right)-\eta$, because $\rho>\delta\left(x_{0}\right)-\eta / 2$. Since $x \in S\left(x_{0}, \sigma\right)$ is arbitrary, the function $\delta$ is 1.s.c. This completes the proof.

TheOREM 3.1. Let $F: X \rightarrow \mathscr{C}(Y)$ be weakly $h^{*}$-u.s.c. Then for every $\varepsilon>0$ there exists a l.s.c. multifunction $G: X \rightarrow \mathscr{C}(Y)$ such that $h($ graph $G$, graph $F) \leq \varepsilon$.

Proof. Let $F: X \rightarrow \mathscr{C}(Y)$ be weakly $h^{*}$-u.s.c. Let $\varepsilon>0$. Denote by $\delta$ the corresponding 1.s.c. function defined in Lemma 3.2. Let $G: X \rightarrow \mathscr{C}(Y)$ be defined by $G(x)=\operatorname{co} F(S(x, \delta(x)))$. By Lemma 3.1, $G$ is 1.s.c.

Since graph $F \subset$ graph $G$, we only need to show that $h^{*}$ (graph $G$, graph $F) \leq \varepsilon$. To this end, let $(\tilde{x}, \tilde{y}) \in \operatorname{graph} G$. Then $\tilde{y} \in G(\tilde{x})$ and so, for some $y_{i} \in F\left(x_{i}\right), \quad x_{i} \in S(\tilde{x}, \delta(\tilde{x}))$, and $\lambda_{i}>0$ (with $\lambda_{1}+\lambda_{2}$ $\left.+\cdots+\lambda_{k}=1\right)$, we have $\tilde{y}=\lambda_{1} y_{1}+\lambda_{2} y_{2}+\cdots+\lambda_{k} y_{k}$. Since $\max \left\{d\left(x_{i}, \tilde{x}\right) \mid i=1,2, \ldots, k\right\}<\delta(\tilde{x})$, there is a $\rho \in \Delta(\tilde{x})$ with $\max \left\{d\left(x_{i}, \tilde{x}\right) \mid i=1,2, \ldots, k\right\}<\rho \leq \delta(\tilde{x})$, and there is an $x^{\prime} \in S(\tilde{x}, \rho)$ such that $h^{*}\left(F(z), F\left(x^{\prime}\right)\right)<\varepsilon$ for every $z \in S(\tilde{x}, \rho)$. Thus $F(z) \subset F\left(x^{\prime}\right)$ $+\varepsilon S$ for every $z \in S(\tilde{x}, \rho)$. In particular, $F\left(x_{i}\right) \subset F\left(x^{\prime}\right)+\varepsilon S$ for $i=$ $1,2, \ldots, k$ and consequently, $\tilde{y} \in F\left(x^{\prime}\right)+\varepsilon S$. Thus $\tilde{y}=y^{\prime}+v$ for some $y^{\prime} \in F\left(x^{\prime}\right)$ and some $v \in \varepsilon S$. Clearly $\left(x^{\prime}, y^{\prime}\right) \in \operatorname{graph} F$ and

$$
e\left((\tilde{x}, \tilde{y}),\left(x^{\prime}, y^{\prime}\right)\right)=\max \left\{d\left(\tilde{x}, x^{\prime}\right),\left\|\tilde{y}-y^{\prime}\right\|\right\}<\max \{\rho, \varepsilon\}=\varepsilon .
$$

This implies $r((\tilde{x}, \tilde{y})$, graph $F)<\varepsilon$. Since $(\tilde{x}, \tilde{y})$ is arbitrary in graph $G$ we have $h^{*}($ graph $G$, graph $F) \leq \varepsilon$. This completes the proof.

TheOREM 3.2. Let $F: X \rightarrow \mathscr{C}(Y)$ be weakly $h^{*}$-u.s.c. Then for every $\varepsilon>0$ there is a continuous single-valued function $g: X \rightarrow Y$ such that $h^{*}($ graph $g$, graph $F) \leq \varepsilon$. 
Proof. By Theorem 3.1 there is a 1.s.c. multifunction $G: X \rightarrow \mathscr{C}(Y)$ such that $h$ (graph $G$, graph $F$ ) $\leq \varepsilon / 2$. By Michael's lemma [25, p. 368] there is a continuous function $g: X \rightarrow Y$ satisfying $r(g(x), G(x))<\varepsilon / 2$ for every $x \in X$. Evidently $h^{*}($ graph $g$, graph $G) \leq \varepsilon / 2$. Hence,

$$
\begin{aligned}
h^{*}(\text { graph } g, \text { graph } F) \leq & h^{*}(\text { graph } g, \text { graph } G) \\
& +h^{*}(\text { graph } G, \operatorname{graph} F) \leq \varepsilon,
\end{aligned}
$$

which completes the proof.

Arguing as in the proof of Theorem 2.1 one can prove the following

TheOREM 3.3. Let $F: X \rightarrow \mathscr{C}(Y)$ be locally convexifying. Then for every $\varepsilon>0$ there exists a continuous single-valued function $g: X \rightarrow Y$ such that $h^{*}($ graph $g$, graph $F) \leq \varepsilon$.

4. Characterizations of some classes of multifunctions. In this section we present some results concerning the characterization of certain classes of multifunctions by means of $h$-continuous approximation.

Let $Y$ be a real normed space. We suppose $Y$ to be endowed with a (translation) invariant metric $\gamma$ (that is $\gamma(u+w, v+w)=\gamma(u, v)$ for every $u, v$, and $w$ in $Y$ ) satisfying the condition:

$$
\gamma(y, 0) \leq\|y\| \text { for every } y \in Y .
$$

This guarantees that the topology on $Y$ induced by $\gamma$ is weaker than the topology induced by the norm. We shall denote by $\gamma_{0}$ the particular invariant metric $\gamma$ on $Y$ which is generated by the norm of $Y$.

For $A, B$ in $2^{Y}$ we set

$$
\begin{aligned}
& h_{\gamma}^{*}(A, B)=\sup \left\{r_{\gamma}(a, B) \mid a \in A\right\}, \\
& h_{\gamma}(A, B)=\max \left\{h_{\gamma}^{*}(A, B), h_{\gamma}^{*}(B, A)\right\} .
\end{aligned}
$$

Here $r_{\gamma}(a, B)=\inf \{\gamma(a, b) \mid b \in B\}$. When $\gamma=\gamma_{0}$ we denote $h_{\gamma}^{*}, h_{\gamma}$ by $h^{*}, h$ respectively. For $A$ in $2^{Y}$ and $\varepsilon>0$ we set $N_{\varepsilon}^{\gamma}(A)=\{y \in$ $\left.Y \mid r_{\gamma}(y, A)<\varepsilon\right\}$.

It is easy to verify that for $A, B, C, D$ in $2^{Y}$ and $\alpha, \alpha_{0} \in \mathbf{R}$ we have:

$$
\begin{aligned}
& h_{\gamma}(A+B, C+D) \leq h_{\gamma}(A, C)+h_{\gamma}(B, D), \\
& h_{\gamma}\left(\alpha A, \alpha_{0} A\right) \rightarrow 0 \text { as } \alpha \rightarrow \alpha_{0} \quad(A \text { bounded in norm }) .
\end{aligned}
$$


Definition 4.1. A multifunction $F: X \rightarrow 2^{Y}$ is called strictly $h_{\gamma}^{*}$-u.s.c. if for every $x \in X$ and for every $\varepsilon>0$ there is a $\delta>0$ such that $h_{\gamma}^{*}(\operatorname{co} F(S(x, \delta)), F(x))<\varepsilon$.

REMARK 4.1. Evidently, each strictly $h_{\gamma}^{*}$-u.s.c. multifunction is also $h_{\gamma}^{*}$-u.s.c. Moreover, a multifunction $F: X \rightarrow \mathscr{C}(Y)$ is strictly $h^{*}$-u.s.c. if and only if $F$ is $h^{*}$-u.s.c.

The symbols $X, Y, \mathscr{C}_{b}(Y), \mathscr{C}_{k}(Y)$ retain the meaning stated in $\S 1$.

THEOREM 4.1. Let $Y$ be a real normed space with an invariant metric $\gamma$ satisfying (4.1). Let $F: X \rightarrow \mathscr{C}_{b}(Y)$. Then the following statements are equivalent:

(a) $F$ is locally bounded (with respect to the norm of $Y$ ), and strictly $h_{\gamma}^{*}$-u.s.c.

(b) There exists a sequence $\left\{G_{n}\right\}$ of $h_{\gamma}$-continuous multifunctions $G_{n}$ : $X \rightarrow \mathscr{C}_{b}(Y)$ such that: (i) for each $n \in \mathbf{N}$ and for every $x \in X$ there is $a$ $\sigma_{n}(x)>0$ such that $F\left(S\left(x, \sigma_{n}(x)\right)\right) \subset G_{n}(x)$, and (ii) $h_{\gamma}\left(G_{n}(x), F(x)\right) \rightarrow 0$ as $n \rightarrow+\infty$.

Proof. (b) $\Rightarrow$ (a). Let $x \in X$. Let $\varepsilon>0$. By (ii) there is an $n_{0} \in \mathbf{N}$ such that $h_{\gamma}\left(G_{n_{0}}(x), F(x)\right)<\varepsilon$. By (i) there is a $\sigma=\sigma_{n_{0}}(x)>0$ such that $F(S(x, \sigma)) \subset G_{n_{0}}(x)$. Hence $\operatorname{co} F(S(x, \sigma)) \subset G_{n_{0}}(x)$ and so $h_{\gamma}^{*}(\operatorname{co} F(S(x, \sigma)), F(x)) \leq h_{\gamma}^{*}\left(G_{n_{0}}(x), F(x)\right)<\varepsilon$, which implies that $F$ is strictly $h_{\gamma}^{*}$-u.s.c. Trivially $F$ is locally bounded. Hence (a) is satisfied.

(a) $\Rightarrow$ (b). Let $n \in \mathbf{N}$. Since $F$ satisfies (a), for every $z \in X$ there is $\delta_{z}^{n}=\delta(z, n), 0<\delta_{z}^{n}<1 / n$, such that the set $F\left(S\left(z, \delta_{z}^{n}\right)\right)$ is bounded (in the norm of $Y)$ and so $\operatorname{co} F\left(S\left(z, \delta_{z}^{n}\right)\right) \in \mathscr{C}_{b}(Y)$. Let $\mathscr{P}_{n}=\left\{p_{z}^{n}\right\}_{z \in X}$ be a partition of unity subordinated to the open covering $\left\{S\left(z, \delta_{z}^{n} / 3\right)\right\}_{z \in X}$ of $X$. Let $G_{n}: X \rightarrow \mathscr{C}_{B}(Y)$ be given by

$$
G_{n}(x)=\sum_{z \in X} p_{z}^{n}(x) G_{z}^{n}, \quad \text { where } G_{z}^{n}=\operatorname{co} F\left(S\left(z, \delta_{z}^{n} / 3\right)\right) .
$$

Clearly $G_{n}$ is well defined. We shall show that $G_{n}$ is $h_{\gamma}$-continuous.

In fact, let $\tilde{x} \in X$ and let $V$ be a neighborhood of $\tilde{x}$ which meets only a finite number of the sets $\operatorname{supp} p_{z}^{n}$. Let $D_{\mathscr{O}_{n}}(V)=\left\{z_{1}, z_{2}, \ldots, z_{k}\right\}$ (the points $z_{i}$ depend on $n$ ). Then for each $x \in V$ we have

$$
h_{\gamma}\left(G_{n}(x), G_{n}(\tilde{x})\right)=h_{\gamma}\left(\sum_{i=1}^{k} p_{i}^{n}(x) G_{z_{\imath}}^{n}, \sum_{i=1}^{k} p_{i}^{n}(\tilde{x}) G_{z_{i}}^{n}\right) \text {. }
$$


From this, by virtue of (4.2), (4.3) and the continuity of the functions $p_{z_{u}}^{n}$, it follows that $h_{\gamma}\left(G_{n}(x), G_{n}(\tilde{x})\right) \rightarrow 0$ as $x \rightarrow \tilde{x}$. Thus $G_{n}$ is $h_{\gamma}$-continuous.

$G_{n}$ satisfies (i). In fact, let $\tilde{x} \in X$ and let $D_{\mathscr{P}_{n}}(\tilde{x})=\left\{z_{1}, z_{2}, \ldots, z_{k}\right\}$. Since $\tilde{x} \in \operatorname{supp} p_{z}^{n} \subset S\left(z_{i}, \delta_{z}^{n} / 3\right)$, there is a $\sigma_{n}(\tilde{x})>0$ such that $S\left(\tilde{x}, \sigma_{n}(\tilde{x})\right) \subset S\left(z_{l}, \delta_{z_{i}}^{n} / 3\right)$ for each $i=1,2, \ldots, k$. Then

$$
F\left(S\left(\tilde{x}, \sigma_{n}(\tilde{x})\right)\right) \subset \sum_{i=1}^{k} p_{z_{l}}^{n}(\tilde{x}) F\left(S\left(\tilde{x}, \sigma_{n}(\tilde{x})\right)\right) \subset \sum_{l=1}^{k} p_{z_{l}}^{n}(\tilde{x}) G_{z_{l}}^{n}=G_{n}(\tilde{x}),
$$

and so (i) is fulfilled.

The sequence $\left\{G_{n}\right\}$ satisfies (ii). To see this, let $\tilde{x} \in X$ and let $\varepsilon>0$. Since $F$ is strictly $h_{\gamma}^{*}$-u.s.c., there is a $\sigma=\sigma(\tilde{X}, \varepsilon)>0$ such that

$$
h_{\gamma}^{*}(\operatorname{co} F(S(\tilde{x}, \sigma)), F(\tilde{x}))<\varepsilon .
$$

Fix an integer $n_{0} \geq 1 / \sigma$ and let $n \geq n_{0}$ be any. Let $D_{\mathscr{P}_{n}}(\tilde{x})=$ $\left\{z_{1}, z_{2}, \ldots, z_{k}\right\}$ (the points $z_{i}$ depend on $n$ ). For each $u \in S\left(z_{l}, \delta_{z_{l}}^{n} / 3\right.$ ) we have

$$
d(u, \tilde{x}) \leq d\left(u, z_{i}\right)+d\left(z_{i}, \tilde{x}\right)<\delta_{z_{i}}^{n} / 3+\delta_{z_{i}}^{n} / 3<2 /(3 n)<\sigma,
$$

and so $S\left(z_{i}, \delta_{z_{i}}^{n} / 3\right) \subset S(\tilde{x}, \sigma)$. Then, we have

$$
G_{n}(\tilde{x})=\sum_{i=1}^{k} p_{z_{i}}^{n}(\tilde{x}) G_{z_{i}}^{n} \subset \sum_{i=1}^{k} p_{z_{i}}^{n}(\tilde{x}) \operatorname{co} F(S(\tilde{x}, \sigma))=\operatorname{co} F(S(\tilde{x}, \sigma)) .
$$

The above inclusion and (4.4) imply $h_{\gamma}^{*}\left(G_{n}(\tilde{x}), F(\tilde{x})\right)<\varepsilon$, if $n \geq n_{0}$. By (i), $h_{\gamma}^{*}\left(F(\tilde{x}), G_{n}(\tilde{x})\right)=0$. Thus $h_{\gamma}\left(G_{n}(\tilde{x}), F(\tilde{x})\right)<\varepsilon$, if $n \geq n_{0}$. This completes the proof.

Theorem 4.2. Let $F: X \rightarrow \mathscr{C}_{k}\left(\mathbf{R}^{q}\right)$ be $K$-closed. Then the following statements are equivalent:

(a) $F$ is $h^{*}$-u.s.c.

(b) $F$ is locally bounded and locally convexifying.

(c) There is a sequence $\left\{G_{n}\right\}$ of h-continuous multifunctions $G_{n}: X \rightarrow$ $\mathscr{C}_{k}\left(\mathbf{R}^{q}\right)$ satisfying the conditions (i), (ii), and (iii) of Theorem 2.3.

(d) There is a sequence $\left\{G_{n}\right\}$ of h-continuous multifunctions $G_{n}: X \rightarrow$ $\mathscr{C}_{k}\left(\mathbf{R}^{q}\right)$ satisfying, in addition to the conditions (i) and (ii) of Theorem 2.3, the following one: (iii)' for each $x \in X, h\left(G_{n}(x), F(x)\right) \rightarrow 0$ as $n \rightarrow+\infty$.

Proof. Indeed, (a) $\Rightarrow$ (b) is obvious, (b) $\Rightarrow$ (c) follows from Theorem 2.3, while (d) $\Rightarrow$ (a) is shown in [8] (see the proof of Theorem 4.5). Let us prove that (c) $\Rightarrow(\mathrm{d})$. To this end, suppose that $\left\{G_{n}\right\}$ satisfies the conditions stated in (c). We claim that $\left\{G_{n}\right\}$ satisfies also (iii'). Suppose the 
contrary. Then there exist $\tilde{x} \in X, \varepsilon>0$, and a subsequence, $\left\{\tilde{G}_{n}\right\}$ say, of $\left\{G_{n}\right\}$ such that $h\left(\tilde{G}_{n}(\tilde{x}), F(\tilde{x})\right)>\varepsilon$ for every $n \in \mathbf{N}$. For each $n \in \mathbf{N}$ take $y_{n} \in \tilde{G}_{n}(\tilde{x})$ such that $r\left(y_{n}, F(\tilde{x})\right)>\varepsilon$. Since $\left\{y_{n}\right\}$ is contained in the compact set $\tilde{G}_{1}(\tilde{x})$, we assume (without loss of generality) that $y_{n} \rightarrow \tilde{y} \in$ $\tilde{G}_{1}(\tilde{x})$. Thus we have $r(\tilde{y}, F(\tilde{x})) \geq \varepsilon$. Since $\left(\tilde{x}, y_{n}\right) \in$ graph $\tilde{G}_{n}$ and (from the hypothesis) $h$ (graph $\tilde{G}_{n}$, graph $F$ ) $\rightarrow 0$ as $n \rightarrow+\infty$, there is a sequence $\left\{\left(x_{n}^{\prime}, y_{n}^{\prime}\right)\right\} \subset$ graph $F$ such that $x_{n}^{\prime} \rightarrow \tilde{x}$ and $y_{n}^{\prime} \rightarrow \tilde{y}$. But graph $F$ is closed, and so $\tilde{y} \in F(\tilde{x})$, a contradiction. This completes the proof.

5. Some applications. In this section we show that an $h^{*}$-u.s.c. (resp. $h^{*}$-1.s.c.) multifunction $F: X \rightarrow \mathscr{C}_{b}(Y)$ is $h$-continuous except at points of a first category set. This is proved using $h$-continuous approximations to $F$. Results of such type have been established, under different hypotheses on $F$, by Hill [15], Kuratowski [22], Choquet [7], Fort [12], and Kenderov [20, 21]. As an application of Theorem 5.1 below, we present an alternative proof of Kenderov's theorem [18] stating that a maximal monotone operator is almost everywhere single-valued. For related results and further generalizations see [19], [14], [30].

THEOREM 5.1. Let $X$ be a metric space. Let $Y$ be a real normed space with an invariant metric $\gamma$ satisfying (4.1). Let $F: X \rightarrow \mathscr{C}_{b}(Y)$ be locally bounded (with respect to the norm of $Y$ ) and strictly $h_{\gamma}^{*}$-u.s.c. Then there exists a Baire first category set $X_{0} \subset X$ such that $F$ restricted to $X \backslash X_{0}$ is $h_{\gamma}$-continuous.

Proof. By Theorem 4.1, there is a sequence $\left\{G_{n}\right\}$ of $h_{\gamma}$-continuous multifunctions $G_{n}: X \rightarrow \mathscr{C}_{b}(Y)$ satisfying at each $x \in X$ the properties: $G_{n}(x) \supset F(x)(n \in \mathbf{N})$, and $h_{\gamma}\left(G_{n}(x), F(x)\right) \rightarrow 0$ as $n \rightarrow \infty$. For $n \in \mathbf{N}$, define $\lambda_{n}: X \rightarrow \mathbf{R}$ by $\lambda_{n}(x)=h_{\gamma}\left(G_{n}(x), F(x)\right)$. We claim that $\lambda_{n}$ is a 1.s.c. function. Evidently $\lambda_{n}(x)=h_{\gamma}^{*}\left(G_{n}(x), F(x)\right)$, because $F(x) \subset$ $G_{n}(x)$. Let $x_{0} \in X$ and let $\eta>0$. Taking into account the $h_{\gamma}$-continuity of $G_{n}$, the fact that $G_{n}(x) \subset N_{\lambda_{n}(x)+\eta}^{\gamma}(F(x))$, and the $h_{\gamma}^{*}$-u.s.c. of $F$, we have

$$
G_{n}\left(x_{0}\right) \subset N_{\eta}^{\gamma}\left(G_{n}(x)\right) \subset N_{\lambda_{n}(X)+2 \eta}^{\gamma}(F(x)) \subset N_{\lambda_{n}(x)+3 \eta}^{\gamma}\left(F\left(x_{0}\right)\right),
$$

for each $x$ in some neighborhood $U$ of $x_{0}$. Consequently, $\lambda_{n}\left(x_{0}\right) \leq \lambda_{n}(x)$ $+3 \eta$ for each $x \in U$. Hence $\lambda_{n}$ is 1.s.c. Thus there is a Baire first category set $X_{n} \subset X$, such that $\lambda_{n}$ restricted to $X \backslash X_{n}$, is continuous. Set $X_{0}=\cup_{n=1}^{\infty} X_{n}$. Evidently $X_{0}$ is of the first category in $X$. Suppose $X \backslash X_{0}$ $\neq \varnothing$ (otherwise there is nothing to prove). Clearly each $\lambda_{n}$ is continuous 
on $X \backslash X_{0}$, and at each point $x \in X \backslash X_{0}$ we have $\lambda_{n}(x) \rightarrow 0$ as $n \rightarrow+\infty$.

We are going to see that $F$ restricted to $X \backslash X_{0}$ is $h_{\gamma}$-continuous. In fact, let $x_{0} \in X \backslash X_{0}$ and let $\varepsilon>0$. Choose $n_{0} \in \mathbf{N}$ such that $\lambda_{n_{0}}\left(x_{0}\right)<$ $\varepsilon / 4$. Take $\delta>0$ so that $x \in S\left(x_{0}, \delta\right) \backslash X_{0}$ implies $\lambda_{n_{0}}(x)<\varepsilon / 2$ and $h_{\gamma}\left(G_{n_{0}}(x), G_{n_{0}}\left(x_{0}\right)\right)<\varepsilon / 4$. Then for each $x \in S\left(x_{0}, \delta\right) \backslash X_{0}$ we have

$$
\begin{aligned}
h_{\gamma}\left(F(x), F\left(x_{0}\right)\right) \leq & h_{\gamma}\left(F(x), G_{n_{0}}(x)\right)+h_{\gamma}\left(G_{n_{0}}(x), G_{n_{0}}\left(x_{0}\right)\right) \\
& +h_{\gamma}\left(G_{n_{0}}\left(x_{0}\right), F\left(x_{0}\right)\right)<\lambda_{n_{0}}(x)+\varepsilon / 4+\lambda_{n_{0}}\left(x_{0}\right) \\
< & \varepsilon / 2+\varepsilon / 4+\varepsilon / 4=\varepsilon,
\end{aligned}
$$

and so the statement of the theorem is proved.

RemarK 5.1. Let $F: X \rightarrow \mathscr{C}_{b}(Y)$ be $h^{*}$-u.s.c. By Remark 4.1 and Theorem 5.1 it follows that there exists a Baire first category set $X_{0} \subset X$ such that $F$ restricted to $X \backslash X_{0}$ is $h$-continuous. Observe that the set $X \backslash X_{0}$ is certainly nonempty and dense in $X$, if $X$ is a Baire space.

Remark 5.2. Let $F: X \rightarrow \mathscr{C}_{b}(Y)$. Define $\tilde{F}: X \rightarrow \mathscr{C}_{b}(Y)$ by $\tilde{F}(x)$ $=\overline{F(x)+S}$. Then $F$ is $h^{*}$-1.s.c. (resp. $h^{*}$-u.s.c.) if and only if $\tilde{F}$ is $h^{*}$-1.s.c. (resp. $h^{*}$-u.s.c.). Evidently, $\tilde{F}$ is $h^{*}$-1.s.c. if $F$ is so. Conversely, suppose that $\tilde{F}$ is $h^{*}$-1.s.c. Let $x_{0} \in X$ and $\varepsilon>0$. Then for each $x$ in a neighborhood $U$ of $x_{0}$ we have

$$
F\left(x_{0}\right)+S \subset \tilde{F}\left(x_{0}\right) \subset \tilde{F}(x)+\varepsilon S=\overline{F(x)+\varepsilon S}+S .
$$

Hence, by Rådström's lemma [27], $F\left(x_{0}\right) \subset \overline{F(x)+\varepsilon S}$ for each $x \in U$, from which the $h^{*}$-l.s.c. of $F$ follows at once. By a similar argument one shows that $F$ is $h^{*}$-u.s.c. if and only if $\tilde{F}$ is so.

TheOREM 5.2. Let F: $X \rightarrow \mathscr{C}_{b}(Y)$ be $h^{*}$-l.s.c. Then there exists a Baire first category set $X_{0} \subset X$ such that $F$ restricted to $X \backslash X_{0}$ is h-continuous.

Proof. In view of Remark 5.2, it is sufficient to show that there is a set $X_{0} \subset X$ of the Baire first category such that $\tilde{F}$ restricted to $X \backslash X_{0}$ is $h$-continuous. Indeed, by [8, Proposition 3.4] there is a sequence $\left\{G_{n}\right\}$ of $h$-continuous multifunctions $G_{n}: X \rightarrow \mathscr{C}_{b}(Y)$ satisfying at each $x \in X$ the properties: $G_{n}(x) \subset \tilde{F}(x)(n \in \mathbf{N})$, and $h\left(G_{n}(x), \tilde{F}(x)\right) \rightarrow 0$ as $n \rightarrow+\infty$. For each $n \in \mathbf{N}$ define $\lambda_{n}: X \rightarrow \mathbf{R}$ by $\lambda_{n}(x)=h\left(\tilde{F}(x), G_{n}(x)\right)$. We claim that $\lambda_{n}$ is a 1.s.c. function. Evidently $\lambda_{n}(x)=h^{*}\left(\tilde{F}(x), G_{n}(x)\right)$, because 
$G_{n}(x) \subset \tilde{F}(x)$. Let $x_{0} \in X$ and let $\eta>0$. By virtue of the $h^{*}$-1.s.c. of $\tilde{F}$, the fact that $\tilde{F}(x) \subset G_{n}(x)+\left(\lambda_{n}(x)+\eta\right) S$, and the $h$-continuity of $G_{n}$ we have

$$
\begin{aligned}
\tilde{F}\left(x_{0}\right) & \subset \tilde{F}(x)+\eta S \subset G_{n}(x)+\left(\lambda_{n}(x)+2 \eta\right) S \\
& \subset G_{n}\left(x_{0}\right)+\left(\lambda_{n}(x)+3 \eta\right) S
\end{aligned}
$$

for every $x$ in some neighborhood $U$ of $x_{0}$. Consequently $\lambda_{n}\left(x_{0}\right) \leq \lambda_{n}(x)$ $+3 \eta$ for every $x \in U$. Hence $\lambda_{n}$ is l.s.c. The end of the proof is like that of Theorem 5.1.

From now on, $Y$ will denote a separable real Banach space and $Y^{*}$ its (topological) conjugate. We denote the pairing between $y^{*}$ in $Y^{*}$ and $y$ in $Y$ by $\left\langle y, y^{*}\right\rangle$. We suppose that $Y$ is endowed with the norm topology.

A multi-valued mapping $F: D(F) \rightarrow 2^{Y^{*}}$ with domain $D(F) \subset Y$ $(D(F) \neq \varnothing)$ is said to be a monotone operator if $\left\langle x-y, x^{*}-y^{*}\right\rangle \geq 0$ for all $x, y \in D(F)$ and all $x^{*} \in F(x)$ and $y^{*} \in F(y)$. It is called a maximal monotone operator if, in addition, the graph of $F$ is not properly contained in the graph of any other monotone operator $G: D(G) \rightarrow 2^{Y^{*}}$ with $D(G) \subset Y$.

Let $\left\{x_{n}\right\}$ be a countable dense subset of $Y$. For $x^{*}, y^{*} \in Y^{*}$ we set

$$
\gamma\left(x^{*}, y^{*}\right)=\sum_{n=1}^{\infty} \frac{1}{2^{n+\left\|x_{n}\right\|}} \frac{\left|\left\langle x_{n}, x^{*}-y^{*}\right\rangle\right|}{1+\left|\left\langle x_{n}, x^{*}-y^{*}\right\rangle\right|} .
$$

It is not difficult to prove that $\gamma$ is an invariant metric on $Y^{*}$. Moreover,

$$
\gamma\left(y^{*}, 0\right) \leq \sum_{n=1}^{\infty} \frac{1}{2^{n+\left\|x_{n}\right\|}} \frac{\left\|x_{n}\right\|\left\|y^{*}\right\|}{1+\left\|x_{n}\right\|\left\|y^{*}\right\|} \leq \sum_{n=1}^{\infty} \frac{\left\|x_{n}\right\|\left\|y^{*}\right\|}{2^{n+\left\|x_{n}\right\|}} \leq\left\|y^{*}\right\|,
$$

thus $\gamma$ satisfies (4.1) (with $Y^{*}$ in the place of $Y$ ).

Let $B^{*}=\left\{y^{*} \in Y^{*} \mid\left\|y^{*}\right\| \leq 1\right\}$. Let $r>0$. On the set $r B^{*}$ we shall consider the topology, denoted $\tau_{\gamma}$, which is generated by the metric $\gamma$, and the relative $\sigma\left(Y^{*}, Y\right)$ topology, denoted $\tau_{\sigma}$. Using the argument of [10, Theorem V. 5.1, p. 426] one can prove that the topologies $\tau_{\gamma}$ and $\tau_{\sigma}$ for $r B^{*}$ are identical.

LemMA 5.1. Let $G: X \rightarrow 2^{Y^{*}}$ ( $X$ a metric space) satisfy the hypotheses: (i) $G(x) \subset r B^{*}(r>0)$ for every $x \in X$; (ii) $G(x)$ is convex and $\sigma\left(Y^{*}, Y\right)$ closed, for every $x \in X$; (iii) $G$ is u.s.c. from $X$ to $2^{Y^{*}}$, where $Y^{*}$ is given the $\sigma\left(Y^{*}, Y\right)$ topology. Then $G$ is strictly $h_{\gamma}^{*}$-u.s.c. 
Proof. By Alaoglu's theorem the space $r B^{*}$ is $\tau_{\sigma}$ compact. Since $\tau_{\sigma}=\tau_{\gamma}$ it follows that $r B^{*}$ is a $\tau_{\gamma}$ compact metric space. Suppose that $G$ satisfies the hypotheses of the lemma but is not strictly $h_{\gamma}^{*}$-u.s.c. Then there exist an $x_{0} \in X$, an $\varepsilon>0$, and a sequence $\left\{\delta_{n}\right\}$ of positive numbers $\delta_{n}$ converging to zero such that $h_{\gamma}^{*}\left(\operatorname{co} G\left(S\left(x_{0}, \delta_{n}\right)\right), G\left(x_{0}\right)\right)>\varepsilon$ for every $n \in \mathbf{N}$. Take $y_{n}^{*} \in \operatorname{co} G\left(S\left(x_{0}, \delta_{n}\right)\right)$ such that $r_{\gamma}\left(y_{n}^{*}, G\left(x_{0}\right)\right)>\varepsilon, n \in \mathbf{N}$. Passing to a subsequence, we suppose that $y_{n}^{*} \rightarrow y^{*} \in r B^{*}$ (in the metric $\gamma)$. Hence $r_{\gamma}\left(y^{*}, G\left(x_{0}\right)\right) \geq \varepsilon$. But in the space $Y^{*}$ with the $\sigma\left(Y^{*}, Y\right)$ topology, the set $G\left(x_{0}\right)$ is closed convex and $y_{0}^{*} \notin G\left(x_{0}\right)$. Then by Hahn-Banach's theorem, there is a continuous linear functional $\varphi: Y^{*} \rightarrow$ $\mathbf{R}$ and there exist constants $c$ and $\theta, \theta>0$, such that

$$
\begin{aligned}
& y_{0}^{*} \in U=\left\{y^{*} \in Y^{*} \mid\left\langle y^{*}, \varphi\right\rangle>c\right\}, \\
& G\left(x_{0}\right) \subset V=\left\{y^{*} \in Y^{*} \mid\left\langle y^{*}, \varphi\right\rangle<c-\theta\right\} .
\end{aligned}
$$

Evidently $y_{n}^{*} \in U \cap r B^{*}$, provided $n$ is large enough, say $n \geq k$. On the other hand, the set $\{x \in X \mid G(x) \subset V\}$ is a neighborhood of $x_{0}$, because $V$ is $\sigma\left(Y^{*}, Y\right)$ open and $G$ is u.s.c. (see Remark 1.10). Hence, for some $n \geq k$ we have $G\left(S\left(x_{0}, \delta_{n}\right)\right) \subset V$. Therefore $y_{n}^{*} \in U \cap V$, a contradiction. This completes the proof.

Lemma 5.2. Let $G: U \rightarrow 2^{Y^{*}}$ be a monotone operator defined on a nonempty open subset $U$ of $Y$. Suppose that: (i) $G(x) \subset r B^{*}(r>0)$ for every $x \in U$; (ii) $G(x)$ is $\sigma\left(Y^{*}, Y\right)$ closed for every $x \in U$; (iii) there is a dense subset $\tilde{U}$ of $U$ such that the restriction $\tilde{G}$ of $G$ to $\tilde{U}$ is $h_{\gamma}$-continuous. Then $\tilde{G}$ is single-valued and demicontinuous (i.e. continuous as a single-valued mapping from $\tilde{U}$ with the relative norm topology, to $Y^{*}$ with the $\sigma\left(Y^{*}, Y\right)$ topology).

Proof. Observe that the $h_{\gamma}$-continuous multifunction $\tilde{G}$ from $\tilde{U}$ to $2^{r B^{*}}$ takes on $\tau_{\gamma}$ compact values. Consequently by Remark 1.9, $\tilde{G}$ is continuous if $r B^{*}$ is assigned the $\tau_{\gamma}$ topology or, equivalently, the $\tau_{\sigma}$ topology.

Arguing as in [18] we shall prove that $\tilde{G}$ is single-valued. In the contrary case, there exist an $x_{0} \in \tilde{U}$ and $u_{0}^{*}, v_{0}^{*} \in \tilde{G}\left(x_{0}\right)$ such that $u_{0} \neq v_{0}^{*}$. Clearly for some $c \in U$ we have $\left|\left\langle c, v_{0}^{*}-u_{0}^{*}\right\rangle\right|>0$. Without loss of generality we can assume that $\eta=\left\langle c, v_{0}^{*}-u_{0}^{*}\right\rangle>0$. For $n$ large enough, say $n \geq n_{0}, c_{n}=x_{0}+(1 / n) c$ lies in $U$. Since $\tilde{U}$ is a dense subset of $U$, there is a sequence $\left\{x_{n}\right\} \subset \tilde{U}$ satisfying $\left\|x_{n}-c_{n}\right\|<1 / n^{2}, n \geq n_{0}$. 
Let $V=r B^{*} \cap\left\{y^{*} \in Y^{*}||\left\langle c, y^{*}-u_{0}^{*}\right\rangle \mid<\eta / 2\right\}$ and observe that $V$ is $\tau_{\sigma}$ open, and $\tilde{G}\left(x_{0}\right) \cap V \neq \varnothing$. By the continuity of $\tilde{G}$ the set $\{x \in$ $\tilde{U} \mid \tilde{G}(x) \cap V \neq \varnothing\}$ is a neighborhood of $x_{0}$ in $\tilde{U}$. Since $x_{n} \rightarrow x_{0}$, there is an $n_{1} \geq n_{0}$ such that $\tilde{G}\left(x_{n}\right) \cap V \neq \varnothing$ for every $n \geq n_{1}$. Let $y_{n}^{*} \in \tilde{G}\left(x_{n}\right)$ $\cap V\left(n \geq n_{1}\right)$. We have

$$
\begin{aligned}
0 & \leq\left\langle x_{n}-x_{0}, y_{n}^{*}-v_{0}^{*}\right\rangle \\
& =\left\langle x_{n}-c_{n}, y_{n}^{*}-v_{0}^{*}\right\rangle+\frac{1}{n}\left\langle c, y_{n}^{*}-u_{0}^{*}\right\rangle+\frac{1}{n}\left\langle c, u_{0}^{*}-v_{0}^{*}\right\rangle \\
& \leq\left\|x_{n}-c_{n}\right\|\left\|y_{n}^{*}-v_{0}^{*}\right\|+\frac{1}{n} \frac{\eta}{2}-\frac{1}{n} \eta<\frac{1}{n}\left[\frac{2 r}{n}-\frac{\eta}{2}\right] .
\end{aligned}
$$

From this, taking $n$ sufficiently large, a contradiction follows. Hence $\tilde{G}$ is single-valued. It is obvious that $\tilde{G}$ is demicontinuous. This completes the proof.

Theorem 5.3 [18]. Let $Y$ be a separable real Banach space and let $Y^{*}$ be its conjugate. Let $F: D(F) \rightarrow 2^{Y^{*}}$ be a maximal monotone operator. Suppose that $\operatorname{int}(\operatorname{co} D(F)) \neq \varnothing$. Then there exists a residual subset $\tilde{D}$ of int $D(F)$ such that $F$ restricted to $\tilde{D}$ is single-valued and demicontinuous.

Proof. Let $F$ satisfy the hyootheses of the theorem. It is well known [28] that int $D(F)$ is a nonempty convex set whose closure is $\overline{D(F)}$, and $F$ restricted to int $D(F)$ is locally bounded (in the norm of $Y^{*}$ ). Furthermore (see [3], [18]) for every $x \in$ int $D(F), F(x)$ is convex and $\sigma\left(Y^{*}, Y\right)$ closed, and $F$ restricted to int $D(F)$ is u.s.c. as a multifunction from int $D(F)$ to $2^{Y^{*}}$, where $Y^{*}$ is assigned the $\sigma\left(Y^{*}, Y\right)$ topology.

For each $n \in \mathbf{N}$, set $U_{n}=\left\{x \in \operatorname{int} D(F) \mid F(S(x, \sigma(x))) \subset n B^{*}\right.$, for some $\sigma(x)>0\}$. Clearly the sets $U_{n}$ are open and $U_{1} \subset U_{2} \subset \cdots$. Moreover for $n$ large enough, say $n \geq k$, each $U_{n}$ is nonempty and $\bigcup_{n \geq k} U_{n}=$ int $D(F)$. The restriction of $F$ to $U_{n}$ satisfies the hypotheses of Lemma 5.1 (with $X=U_{n}$ and $r=n$ ) and so is strictly $h_{\gamma}^{*}$-u.s.c. By Theorem 5.1 (with $Y^{*}$ in the place of $Y$ ) there is a set $Z_{n} \subset U_{n}$ of the first Baire category in $U_{n}$ (and so with dense complement $\tilde{U}_{n}=U_{n} \backslash Z_{n}$ ) such that the restriction of $F$ to $\tilde{U}_{n}$ is $h_{\gamma}$-continuous. By virtue of Lemma 5.2, $F$ restricted to $U_{n}$ is single-valued and demicontinuous. Set $Z_{0}=\cup_{n \geq k} Z_{n}$, and $\tilde{D}=$ int $D(F) \backslash Z_{0}$. Evidently $\tilde{D}$ is a residual subset of int $D(F)$ and $F$ restricted to $\tilde{D}$ is single-valued and demicontinuous. This completes the proof. 


\section{REFERENCES}

[1] C. Berge, Espaces topologiques, fonctions multivoques, Dunod, Paris, 1966.

[2] Yu. G. Borisovich, B. D. Gel'man, A. D. Myshkis and V. V. Obukhovskii, Topological methods in the fixed point theory of multi-valued maps (Russian), Uspehi Mat. Nauk, 35 (1980), 59-126.

[3] F. E. Browder, Multi-valued monotone nonlinear mappings and duality mappings in Banach spaces, Trans. Amer. Math. Soc., 118 (1965), 338-351.

[4] C. Castaing and M. Valadier, Convex Analysis and Measurable Multifunctions, Lecture Notes in Mathematics, Vol. 580, Springer-Verlag, Berlin, 1977.

[5] A. Cellina, $A$ theorem on the approximation of compact multivalued mappings, Atti Accad. Naz. Lincei Rend. Cl. Sci. Fis. Mat. Natur., (8) (1969), 149-153.

[6] _ A further result on the approximation of set-valued mappings, Atti Accad. Naz. Lincei Rend. Cl. Sci. Fis. Mat. Natur., (8) (1970), 230-234.

[7] G. Choquet, Convergences, Ann. Univ. Grenoble, Sect. Sci. Math. Phys. (N.S.), 23 (1948), 7-112.

[8] F. S. De Blasi, Characterizations of certain classes of semicontinuous multifunctions by continuous approximations, J. Math. Anal. Appl., 106 (1985), 1-18.

[9] J. Dugundji, Topology, Allyn and Bacon, Boston, 1966.

[10] N. Dunford and J. T. Schwartz, Linear Operators I, Interscience, New York, 1957.

[11] M. Edelstein and A. C. Thompson, Some results on nearest points and support properties of convex sets in $c_{0}$, Pacific J. Math., 40 (1972), 553-560.

[12] M. K. Fort, Points of continuity of semi-continuous functions, Public. Mathem. Debrecen, 2 (1951), 100-102.

[13] G. Haddad, Topological properties of the sets of solutions for functional differential inclusions, Nonlinear Anal., 5 (1981), 1349-1366.

[14] R. W. Hansell, J. E. Jayne and P. S. Kenderov, Semi-continuité inférieure générique d'une multiapplication, C. R. Acad. Sc. Paris Sér. I. Math., 296 (1983), 497-500.

[15] L. S. Hill, Properties of certain aggregate functions, Amer. J. Math., 49 (1927), 419-432.

[16] Shui-Hung Hou, On property $(Q)$ and other semicontinuity properties of multifunctions, Pacific J. Math., 103 (1982), 39-56.

[17] M. Hukuhara, Sur l'application semi-continue dont la valeur est un compact convexe, Funkcial. Ekvac., 10 (1967), 43-66.

[18] P. S. Kenderov, Semi-continuity of set-valued monotone mappings, Fund. Math., 87 (1975), 61-69.

[19] _ Multivalued monotone mappings are almost everywhere single-valued, Studia Math., 56 (1976), 199-203.

[20] Points of single-valuedness of multivalued metric projections, C. R. Acad. Bulgare Sci., 29 (1976), 773-775.

[21] Uniqueness on a residual part of best approximation in Banach spaces, Pliska Stud. Math. Bulgar., 1 (1977), 122-127.

[22] C. Kuratowski, Sur les décompositions semi-continues d'espaces metriques compacts, Fund. Math., 11 (1928), 169-186.

[23] A. Lasota and Z. Opial, An approximation theorem for multivalued mappings, Podstawy Sterowania, 1 (1971), 71-75.

[24] J. M. Lasry and R. Robert, Analyse non linéaire multivoque, Cahiers de Mathématiques de la Décision, No. 7611, Centre de Recherche de Mathématiques de la Décision, Université de Paris IX Dauphine.

[25] E. Michael, Continuous selections I, Ann. of Math., (2) 63 (1956), 361-382. 
[26] J. J. Moreau, Multiapplications à retraction finie, Ann. Scuola Norm. Sup. Pisa Cl. Sc., (4) 1 (1974), 169-203.

[27] H. Rådström, An embedding theorem for spaces of convex sets, Proc. Amer. Math. Soc., 3 (1952), 165-169.

[28] R. T. Rockafellar, Local boundedness of nonlinear monotone operators, Michigan Math. J., 16 (1969), 397-407.

[29] J. Von Neumann, A Model of General Economic Equilibrium, Collected works, Vol. VI, Pergamon Press, Oxford, 1963, 29-37.

[30] E. H. Zarantonello, Dense single-valuedness of monotone operators, Israel J. Math., 15 (1973), 158-166.

Received August 11, 1983 and in revised form March 5, 1985.

UNIVERSITA DI ROMA II

VIA D. RAIMONDO

00173 RoMA, ITALY

AND

INSTYTUT MATEMATYKI AGH

AL. MICKIEWICZA 30

30-059 KraKow, Poland 



\section{PACIFIC JOURNAL OF MATHEMATICS EDITORS}

V. S. VARADARAJAN

(Managing Editor)

University of California

Los Angeles, CA 90024

HERBERT CLEMENS

University of Utah

Salt Lake City, UT 84112

R. FINN

Stanford University

Stanford, CA 94305
HERMANN FLASCHKA

University of Arizona

Tucson, AZ 85721

RAMESH A. GANGOLLI

University of Washington

Seattle, WA 98195

VAUGHAN F. R. JONES

University of California

Berkeley, CA 94720

ROBION KIRBY

University of California

Berkeley, CA 94720
C. C. MOORE

University of California

Berkeley, CA 94720

H. SAMELSON

Stanford University

Stanford, CA 94305

HAROLD STARK

University of California, San Diego

La Jolla, CA 92093

\section{ASSOCIATE EDITORS}

R. ARENS

E. F. BECKENBACH

B. H. NEUMANN (1906-1982)
F. WOLF

K. YOSHIDA

\section{SUPPORTING INSTITUTIONS}

UNIVERSITY OF ARIZONA

UNIVERSITY OF BRITISH COLUMBIA

CALIFORNIA INSTITUTE OF TECHNOLOGY

UNIVERSITY OF CALIFORNIA

MONTANA STATE UNIVERSITY

UNIVERSITY OF NEVADA, RENO

NEW MEXICO STATE UNIVERSITY

OREGON STATE UNIVERSITY
UNIVERSITY OF OREGON UNIVERSITY OF SOUTHERN CALIFORNIA

STANFORD UNIVERSITY

UNIVERSITY OF HAWAII

UNIVERSITY OF TOKYO

UNIVERSITY OF UTAH

WASHINGTON STATE UNIVERSITY

UNIVERSITY OF WASHINGTON 


\section{Pacific Journal of Mathematics}

\section{Vol. 123, No. $1 \quad$ March, 1986}

Maria Emilia Alonso García, A note on orderings on algebraic varieties $\ldots \ldots 1$

F. S. De Blasi and Józef Myjak, On continuous approximations for

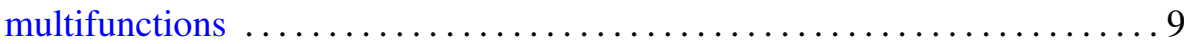

Frank Albert Farris, An intrinsic construction of Fefferman's CR metric . . . 33 Antonio Giambruno, P. Misso and Francisco César Polcino Milies, Derivations with invertible values in rings with involution $\ldots . \ldots . \ldots .47$

Dan Haran and Moshe Jarden, The absolute Galois group of a pseudo real

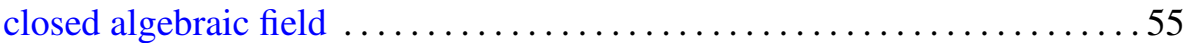

Telemachos E. Hatziafratis, Integral representation formulas on analytic

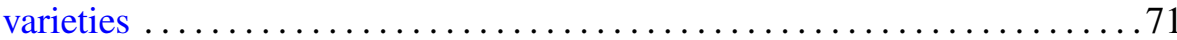

Douglas Austin Hensley, Dirichlet's theorem for the ring of polynomials

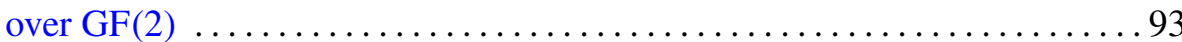

Sofia Kalpazidou, On a problem of Gauss-Kuzmin type for continued

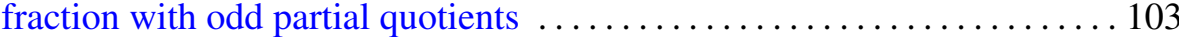

Harvey Bayard Keynes and Mahesh Nerurkar, Ergodicity in affine

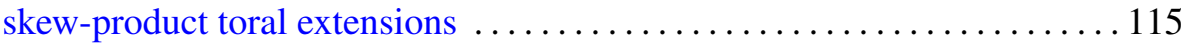

Thomas Landes, Normal structure and the sum-property $\ldots \ldots \ldots \ldots \ldots 127$

Anthony To-Ming Lau and Viktor Losert, Weak*-closed complemented invariant subspaces of $L_{\infty}(G)$ and amenable locally compact groups ...149 Andrew Lelek, Continua of constant distances in span theory . . . . . . . . 161 Dominikus Noll, Sums and products of $B_{r}$ spaces $\ldots \ldots \ldots \ldots \ldots \ldots \ldots \ldots$ Lucimar Nova, Fixed point theorems for some discontinuous operators 189

A. A. S. Perera and Donald Rayl Wilken, On extreme points and support points of the family of starlike functions of order $\alpha$

Massimo A. Picardello, Positive definite functions and $L^{p}$ convolution operators on amalgams ........................

Friedrich Roesler, Squarefree integers in nonlinear sequences ......... 223

Theodore Shifrin, The osculatory behavior of surfaces in $\mathbf{P}^{5}$ 